\title{
A Derivation of the Main Relations of Nonequilibrium Thermodynamics
}

\author{
Vladimir N. Pokrovskii \\ Moscow State University of Economics, Statistics and Informatics, Moscow 119501, Russia \\ Correspondence should be addressed to Vladimir N. Pokrovskii; vpok@comtv.ru
}

Received 9 July 2013; Accepted 5 September 2013

Academic Editors: C. D. Daub, P. Espeau, A. Ghoufi, and P. Trens

Copyright ( 2013 Vladimir N. Pokrovskii. This is an open access article distributed under the Creative Commons Attribution License, which permits unrestricted use, distribution, and reproduction in any medium, provided the original work is properly cited.

The principles of nonequilibrium thermodynamics are discussed, using the concept of internal variables that describe deviations of a thermodynamic system from the equilibrium state. While considering the first law of thermodynamics, work of internal variables is taken into account. It is shown that the requirement that the thermodynamic system cannot fulfil any work via internal variables is equivalent to the conventional formulation of the second law of thermodynamics. These statements, in line with the axioms introducing internal variables can be considered as basic principles of nonequilibrium thermodynamics. While considering stationary nonequilibrium situations close to equilibrium, it is shown that known linear parities between thermodynamic forces and fluxes and also the production of entropy, as a sum of products of thermodynamic forces and fluxes, are consequences of fundamental principles of thermodynamics.

\section{Introduction}

The modern nonequilibrium thermodynamics is formulated [1-3] as a generalisation of equilibrium thermodynamics, as adding some concepts and principles, in particular, the concepts of fluxes and thermodynamic forces, specific for nonequilibrium. Despite many different approaches the problem, reviewed recently by Muschik [4], the extension of equilibrium thermodynamics to non-equilibrium thermodynamics seems to need some justification. We are going to follow the approach $[5,6]$, which exploits additional variables, socalled internal variables, ${ }^{1}$ to describe deviations of a state of thermodynamic system from equilibrium. It can be thought, that this approach allows one to explore the principles of nonequilibrium thermodynamics, providing some justification of the known linear relations and opens opportunities for nonlinear generalizations. We have to note that formulation of the main principles of non-equilibrium thermodynamics in terms of internal variables is disputable, there is, at least, two explicit versions. In particular, one of the approaches [6] takes into consideration only distinctive internal variables, those that can be called [7] complexity internal variables. The other approach, which is followed in this paper, considers all quantities, which describe the deviation of the system from the equilibrium, to be internal variables. In this paper, we are trying to show advantages of our description [7] as compared with the alternative formulation $[4,6]$.

Section 2 begins with a description of a set of variables needed for the depiction of a non-equilibrium state of a thermodynamic system. Further, reproducing partly the previous paper [7], we pay a special attention to formulation of the first and second principles of thermodynamics in terms of internal variables. Dynamics of internal variables is discussed in Section 3. Section 4 is devoted to consideration of stationary states, while it is shown, that, in the areas close to equilibrium, the known linear relation between thermodynamic forces and fluxes and also expression for production of entropy, as a sum of products of fluxes and thermodynamic forces, follow the formulated general principles. Conclusion contains a discussion of the results.

\section{The Main Concepts and Principles}

Let us consider a thermodynamic system, the equilibrium state of which, on definition, is characterized by absolute temperature $^{2} T$ and constitutive variables $x_{1}, x_{2}, \ldots, x_{n}$. The specified constitutive variables determine thermodynamic 
system and define its borders. In the simplest case, a volume $V$, which contains a certain part of substance in a gas or liquid phase, is considered as a constitutive variable.

To define a nonequilibrium state of the same system, one needs, apart from temperature (in this case the concept of temperature ought to be discussed specially [6]) and constitutive variables, in an indefinite number of other variables $\xi_{1}, \xi_{2}, \ldots$. When a limited list of $n$ constitutive variables is fixed, the other variables are called internal variables; in an equilibrium situation there are no internal variables or, otherwise, we can consider values of internal variables whatever their set is, are equal to zero, which corresponds to the agreement that the internal variables describe deviations of the system from the equilibrium. ${ }^{3}$ The internal variables characterize local heterogeneity of system and, consequently, can be named as structural variables also. The thermodynamic system has no predetermined set of internal parameters; they arise, generally speaking, at any influence on the system and, consequently, it is possible to note also that internal variables in the system represent disappearing memory of the past interactions with the environment.

Thus, the state space of a system is defined as $\left(T, x_{1}\right.$, $\left.x_{2}, \ldots x_{n} ; \xi_{1}, \xi_{2} \ldots\right)$, where temperature $T$ is a unique characteristic for both equilibrium and non-equilibrium, and any space deviations from this quantity are considered as internal variables. It is not the unique way of determining the system, but it is convenient to adopt the listed variables for the beginning. Under unchanging conditions, the thermodynamic system is trending to a unique steady macrostate, which is referred to as the equilibrium state. The trend of the system to the equilibrium state means a trend of all internal variables to zero values.

2.1. The First Principle of Thermodynamics. First of all, let us consider the balance of all influences on the system. Taking into account energy coming into the system with fluxes of heat and matter from the environment, $\Delta Q$ and $\Delta N_{j}$, and work of the system ${ }^{4}$ through constitutive variables (work, performed by the system, is considered positive), one can write for a change of total energy $U$ of the system that

$$
\Delta U=\Delta Q-\sum_{i=1}^{n} X_{i} \Delta x_{i}+\sum_{j=1}^{N} \mu_{j} \Delta N_{j} .
$$

This is a conventional form of the balance of energy $[4,6]$.

In line with the total internal energy $U$, which includes possible potential energy of agitated internal variables, internal thermal energy $E$, which does not depend on internal variables, can be introduced [7]. In the equilibrium situations, total energy $U$ coincides with internal energy $E$, but, in the non-equilibrium situations, these quantities are different. ${ }^{5}$ Then, the law of conservation of energy (1) can be rewritten in the form

$$
\begin{aligned}
\Delta E= & \Delta Q-\sum_{i=1}^{n} X_{i} \Delta x_{i}-\sum_{i} \Xi_{i} \Delta \xi_{i} \\
& +\sum_{j=1}^{N} \mu_{j} \Delta N_{j},
\end{aligned}
$$

where work of the system via internal variables is included. This form of the first principle of thermodynamics demonstrates, that the external influences (work via constitutive variables, heat energy and chemical energy of particles) contribute to a change of internal energy $E$ and emerging of internal variables. It is important to take into account the processes of reallocation of energy due to the work of the internal variables in the explicit form.

2.2. The Second Principle of Thermodynamics. The work connected with the internal variables should be considered as an essential element of the description. Internal variables can be agitated by external influences, but the system cannot perform any positive work via internal variables, which means

$$
\sum_{i} \Xi_{i} \Delta \xi_{i} \leq 0 .
$$

This statement, formulated earlier [7], introduces impossibility of the reversion of evolution of the thermodynamic system in time and can be considered as a formulation of the second principle of thermodynamics - the formulation, which is, as it will be shown below, equivalent to the formulation of the principle in terms of entropy (see Section 2.4).

2.3. The Introduction of Entropy. To define thermodynamic quantities as functions of state, that is, functions of the temperature and constitutive variables, one needs in the concept of entropy with help of which a change of internal energy $E$, which does not depend on internal variables on the definition, can be written as the total differential of variables of state:

$$
d E=T d S-\sum_{i=1}^{n} X_{i} d x_{i}
$$

The relation is valid both for reversible and irreversible processes.

Entropy $S$ can be defined here, on a comparison of relations (2) and (4), as a quantity, a change of which is determined by external influences and internal changes:

$$
T d S=\Delta Q-\sum_{i} \Xi_{i} \Delta \xi_{i}+\sum_{j=1}^{K} \mu_{j} \Delta N_{j} .
$$

Remarkably, when the external fluxes are absent, entropy, in virtue of (5), can be considered as a function of internal variables, whereas the change of entropy (5) can be considered as a total differential of the function. To calculate entropy $S$, in this case, one can consider process of changing of the system from a nonequilibrium state with the fixed values of variables $\xi_{1}, \xi_{2}, \ldots \xi_{s}$ to the equilibrium state. The result does not depend on the way of integration, and the difference between values of entropy in equilibrium and nonequilibrium states can be written symbolically as

$$
\begin{aligned}
& S(T, \mathbf{x}, \boldsymbol{\xi})-S(T, \mathbf{x}, 0) \\
& \quad=-\frac{1}{T} \int_{0}^{\xi_{1}, \xi_{2}, \ldots \xi_{s}} \sum_{j} \Xi_{j} d \xi_{j} \leq 0 .
\end{aligned}
$$


Relation (6) can be considered as a definition of entropy of a nonequilibrium state of thermodynamic system. The definition is applicable to any systems, including open systems in situations far from equilibrium.

Note that relation (5) is a generalisation of Prigogine's relation, given by him for the systems with chemical reactions $\left[1\right.$, Equation 3.52]. ${ }^{6}$

2.4. Two Parts of the Entropy Variation. The total change of entropy in the system $d S$, defined by (5), can be split, due to Prigogine [1], into two components:

$$
d S=d_{e} S+d_{i} S
$$

Change of Entropy due to the Fluxes. The component $d_{e} S$ is connected with flows of heat and substances through the boundaries of the system:

$$
d_{e} S=\frac{1}{T}\left(\Delta Q+\sum_{j=1}^{N} \mu_{j} \Delta N_{j}\right),
$$

where $N_{j}$ is a number of molecules of substance $j$ in the system, and $\mu_{j}$ is a chemical potential. The quantity $d_{e} S$ can be both positive (the flux of heat and/or substances into the system) and negative (the flux of heat and/or substances out of the system). In an isolated system, when there are neither heat fluxes nor matter fluxes between the system and environment, $d_{e} S=0$.

The fluxes of heat and substances into the system or from the system appear at absence of balance between the system and environment. As characteristic quantities, real streams of heat and/or substances through borders of the system are fixed. The actual fluxes into thermodynamic system are specified by a problem under consideration, but, in any case, it is possible to define empirically a set of fluxes $J_{1}, J_{2}, \ldots, J_{r}$ which, it is supposed, are given.

It is convenient to write expression (8) for a change, due to external fluxes into the system, of entropy in a standardized form:

$$
\frac{d_{e} S}{d t}=\frac{1}{T} \sum_{j=1}^{r} K_{j} J_{j},
$$

where quantities $K_{j}$ are characteristics of the system, that is, functions of temperature, constitutive variable, and also internal variables.

Production of Entropy. The internal part of the change of entropy $d_{i} S$ is connected with some processes within the system:

$$
d_{i} S=-\frac{1}{T} \sum_{j} \Xi_{j} \Delta \xi_{j}, \quad d_{i} S \geq 0 .
$$

That means, that some internal variables, which cannot be identified in advance and the number of which is not known, appear to be agitated during transition from one macroscopic state to another and then relax according to their internal laws. Under reversible processes, when the situation changes in such a slow way (characteristic time of process $t \gg$ characteristic time of relaxation $\tau_{i}$ ), that all internal variables actually have their equilibrium values, internal production of entropy is equal to zero, $d_{i} S=0$. By virtue of (3), the quantity $d_{i} S$ can be only nonnegative, and this is a conventional expression of the second law of thermodynamics.

From (10), expression for the production of entropy is as follows:

$$
\frac{d_{i} S}{d t}=-\frac{1}{T} \sum_{j} \Xi_{j} \frac{d \xi_{j}}{d t}, \quad \frac{d_{i} S}{d t} \geq 0 .
$$

Entropy increases, when relaxation of internal variables in the system occurs, so that the production of entropy is a manifestation of presence of internal variables, that is a manifestation of an internal complexity of the system.

\section{Dynamics of Internal Variables}

To move further, our speculations should be completed by equations, which allow to determine evolution of internal variables. Let us consider a system in a non-equilibrium state $(T, \mathbf{x}, \boldsymbol{\xi})$ and under external influences, which can be a change of constitutive variables and/or a change of temperature and concentration of substances in the environment. In the case of reversible processes, the external influences are considered to be weak, so that the changes of $T$ and $\mathbf{x}$ follow external influences. In a general case, to describe the actual situation, one has to consider emerging and evolution of the internal variables, which are not influenced by the external forces directly. As an example, we can point to a situation, when temperature of the environment goes up and heat starts to get into the thermodynamic system. In this situation, gradients of temperature, that is, in our interpretation, internal variables are emerging and internal processes appear; the system trends to a new equilibrium, but the state of the system is determined by a play between external influences and internal processes. It is convenient to consider these contributions separately.

3.1. Preferable Values of Internal Variables. Under external influences, the system is found to be, generally speaking, in a non-equilibrium state, and for the description of the situation, one needs on introduction of some distinctive internal variables $\xi$, as was explained, for example, by Kestin [8] and Muschik [9]. A change of entropy of the system $S(T, \mathbf{x}, \boldsymbol{\xi})$ occurs due to changing of arguments of the function and, apart from it, due to an incoming flux of entropy, that is, a contribution defined by expression (9). Similar to the case of isolated systems, when a maximum value of entropy defines the equilibrium state, extreme value of entropy under the presence of external influences defines special points in the state space. These points are defined by a relation obtained by equating total variation of entropy to zero:

$$
\begin{array}{r}
\frac{\partial S}{\partial T} \frac{d T}{d t}+\frac{\partial S}{\partial \mathbf{x}} \frac{d \mathbf{x}}{d t}+\frac{\partial S}{\partial \boldsymbol{\xi}} \frac{d \boldsymbol{\xi}}{d t} \\
+\frac{1}{T} \sum_{j=1}^{r} K_{j}(T, \mathbf{x}, \boldsymbol{\xi}) J_{j}=0 .
\end{array}
$$


The incoming flux of entropy is different, generally speaking, from the flux of entropy, coming out of the environment.

The relation (12) at given temperature, constitutive variables, and external fluxes can be considered as an equation for unknown internal variables, which, in this case, determines preferable values of internal variables $\xi^{\circ}$.

The rate of change of internal variables in a point of quasiequilibrium can be replaced by rate of change of preferable variables (see (16)):

$$
\left.\frac{d \xi}{d t}\right|_{\xi=\xi^{\circ}}=\frac{d \xi^{\circ}}{d t} .
$$

This allows, using also definition of thermodynamic force, to write the equation for preferable values as

$$
\begin{aligned}
\Xi\left(\xi^{\circ}\right) \frac{d \xi^{\circ}}{d t}= & T\left(\frac{\partial S}{\partial T} \frac{d T}{d t}+\frac{\partial S}{\partial \mathbf{x}} \frac{d \mathbf{x}}{d t}\right)_{\xi=\xi^{\circ}} \\
& +\sum_{j=1}^{r} K_{j}\left(T, \mathbf{x}, \xi^{\circ}\right) J_{j} .
\end{aligned}
$$

The preferable values of internal variables, corresponding to the actual values of other variables and external influence, are determined, apparently, by history of the application of external influences.

In general case, (14) cannot unambiguously define preferable values. However, the situation is not so hopeless, when considering special cases. If temperature and constitutive variables of the system do not change and external influences are given by constant fluxes of heat and substances into the system $J_{1}, J_{2}, \ldots, J_{s}$, it is possible to make some assumptions. We consider, that each flux corresponds to the only one of the internal variables, so that the number of distinctive preferable variables is equal to the number of fluxes, that is, $\xi_{l}^{\circ} \neq 0$ at $l=1,2, \ldots, s$ and $\xi_{l}^{\circ}=0$ at $l=s+1, s+2, \ldots$. Taking also the arbitrariness in the fluxes into account, (14) can be reduced to a set of equations:

$$
\frac{d \xi_{i}^{\circ}}{d t}=B_{i} J_{i}, \quad i=1,2, \ldots, s .
$$

Values of factors $B_{i}$ are settled by a choice of fluxes in respect to a choice of internal variables. Quantities $B_{i}$ have only numerical values, when an "appropriate" choice of fluxes in the form of $J_{j} \sim \xi_{j} / \tau_{j}$, where $\tau_{j}$ is a time of relaxation, has been made. By a choice of the internal variables and the fluxes, it is possible to reduce values $B_{i}$ to units, which is accepted further.

3.2. Evolution of Internal Variables. The actual values of the internal variables $\xi_{1}, \xi_{2}, \ldots$ differ from the preferable values. The deviations of internal variables from their current preferable values $\xi_{i}-\xi_{i}^{\circ}, \quad(i=1,2, \ldots)$ determine the trending of the internal variables to a preferable values. The change of internal variables $\xi_{1}, \xi_{2}, \ldots$ is determined by internal laws of movement of particles of the thermodynamic system, so that an equation for a change of the internal variables can be written in the general form:

$$
\begin{aligned}
& \frac{d\left(\xi_{i}-\xi_{i}^{\circ}\right)}{d t} \\
& =-R_{i j}\left(T, x_{1}, x_{2}, \ldots, x_{n} ; \xi_{1}, \xi_{2}, \ldots ; \xi_{1}^{\circ}, \xi_{2}^{\circ}, \ldots\right) \\
& \quad \times\left(\xi_{j}-\xi_{j}^{\circ}\right), \quad i=1,2, \ldots .
\end{aligned}
$$

This equation describes relaxation of internal variables to preferable values $\xi^{\circ}$, which are defined by (14), in general form, or, for the simple situation, by (15). The right-hand side of (16) describes change of the variables, according to internal laws of the system, the external influences are presented via the preferable values. The sign "minus" is chosen for convenience: matrix $R_{i j}$ in the situations close to equilibrium (at $\left.\xi_{i}^{\circ}=0, i=1,2, \ldots\right)$ is positive definite in many simple cases. ${ }^{7}$

Let us notice, that the internal variables are depending generally on the space coordinates $\xi_{i}=\xi_{i}(t, x, y, z)$, so that the equations for change of internal variables can contain also terms (omitted here) for the description of processes of diffusion of internal variables. Moreover, the equation of evolution of internal variables should include random forces. Each internal variable can be presented as a sum of the regular (averaged in some way) and random components. Here the regular components of variables are considered only. Discussion of random components, also as well as fluctuations of thermodynamic variables, is omitted. For expansion of the description, it is possible to use the mathematical apparatus of stochastic nonlinear nonequilibrium thermodynamics described by Stratonovich [10].

\section{The Stationary Nonequilibrium States}

The situation is being simplified in a steady-state case, when a set of constant fluxes $J_{1}, J_{2}, \ldots, J_{s}$ is fixed. It is assumed that the number of fluxes corresponds to the number of complexity variables, which have distinctive constant values $\xi_{1}^{\circ}, \xi_{2}^{\circ}, \ldots, \xi_{s}^{\circ}$. Apart of it, an indefinite number of some other internal variables $\xi_{s+1}, \xi_{s+2}, \ldots$ can appear, and thermodynamic characteristics of the system are functions of the all internal variables. The non-equilibrium stationary states of thermodynamic system represent special interest, and it is remarkable that these states, also as equilibrium states of the thermodynamic systems, can be considered in a general way.

4.1. Dynamics of Internal Variables. To formulate the dynamic equation for internal variables for a stationary state, we take (16) with definition of the derivative of preferable variables (15), in which the quantities $B_{j}$ is settled to be equal unities. This allows one to write the equation for dynamics of internal variables near a steady-state point:

$$
\begin{array}{r}
\frac{d \xi_{i}}{d t}=J_{i}-R_{i l}\left(\xi_{l}-\xi_{l}^{\circ}\right)-\frac{\partial R_{i k}}{\partial \xi_{l}}\left(\xi_{k}-\xi_{k}^{\circ}\right)\left(\xi_{l}-\xi_{l}^{\circ}\right)+\cdots, \\
i=1,2, \ldots, s, s+1, s+2, \ldots,
\end{array}
$$


where a matrix $R_{i l}$ and its derivatives are fixed in a considered preferable point. The matrix $R_{i j}$ depends on temperature $T$ and constitutive variables $x_{1}, x_{2}, \ldots, x_{n}$ and assumed to be positive definite, that is to have positive eigenvalues. ${ }^{8}$ Let us remind, that the preferable state is defined in such a way, that $\xi_{l}^{\circ} \neq 0$ at $l=1,2, \ldots, s$ and $\xi_{l}^{\circ}=0$ at $l=s+1, s+2, \ldots$.

In stationary state, values of preferable variables are constant; however it is reached by balance of processes of relaxation and permanent excitation of internal variables by external fluxes. Considering the processes of relaxation and excitation in a stationary situation independent, instead of (17), one can write two equations, in linear approximation:

$$
\frac{d \xi_{i}}{d t}=-R_{i j} \xi_{j}, \quad J_{i}=-R_{i j} \xi_{j}^{\circ}, \quad i=1,2, \ldots, s .
$$

The rate of change of internal variables $\xi_{j}$ depends on deviations of values of internal variables from the equilibrium values, but preferable values $\xi_{j}^{\circ}$, which, on the assumption, are close to $\xi_{j}$, are determined by fluxes. An analysis of an empirical situation specifies the second set of equations from (18), thus, establishing the set of internal complexity variables.

4.2. Entropy Near a Stationary State. In situations close to equilibrium, an expansion of entropy into series with respect to internal variables contains no terms of the first order, in the simplest approach:

$$
\begin{gathered}
S(\xi)=S(0)-\frac{1}{2} \sum_{i, j} S_{i j} \xi_{i} \xi_{j}+\cdots, \\
S_{i j}=-\left(\frac{\partial^{2} S}{\partial \xi_{i} \partial \xi_{j}}\right)_{T, \mathbf{x}, \xi=0} .
\end{gathered}
$$

By virtue of (6), value of entropy of the system in a nonequilibrium state is less than value of entropy of the same system in the equilibrium state, so that one has to consider matrix $S$ to be nonnegative determined. Components of the matrix $S$ are functions of temperature and constitutive variables.

An expansion of entropy of the thermodynamic system near a preferable (stationary) point begins with linear terms:

$$
\begin{aligned}
S(\boldsymbol{\xi})= & S\left(\xi^{\circ}\right)-\sum_{j=1}^{s} S_{j}\left(\xi_{j}-\xi_{j}^{\circ}\right) \\
& -\frac{1}{2} \sum_{i, j} S_{i j}\left(\xi_{i}-\xi_{i}^{\circ}\right)\left(\xi_{j}-\xi_{j}^{\circ}\right)+\cdots, \\
S_{j}= & -\left(\frac{\partial S}{\partial \xi_{j}}\right)_{T, \mathbf{x}, \xi^{\circ}}, \quad j=1,2, \ldots, s, \\
S_{i j}= & -\left(\frac{\partial^{2} S}{\partial \xi_{i} \partial \xi_{j}}\right)_{T, \mathbf{x}, \xi^{\circ}}, \quad i, j=1,2, \ldots
\end{aligned}
$$

It is taken into account that, apart from of the complexity internal variables $\xi_{1}, \xi_{2}, \ldots, \xi_{s}$, there is a set of internal variables with numbers $s+1, s+2, \ldots$, that describe deviations of the system from the stationary state. Matrixes $S_{j}$ and $S_{l k}$ are calculated not in equilibrium point, as in expression (19), but in the stationary point and depend, apart from temperature and constitutive variables, on the complexity internal variables.

As a function of the internal complexity variables, entropy has no features, which would allow to characterize the stationary state. Considering the variables of complexity to be fixed, expansion of entropy, as a function of all other possible internal variables, is reduced to a form:

$$
\begin{gathered}
S(\xi)=S\left(\xi^{\circ}\right)-\frac{1}{2} \sum_{i, j=s+1}^{\infty} S_{i j} \xi_{i} \xi_{j}+\cdots, \\
S_{i j}=-\left(\frac{\partial^{2} S}{\partial \xi_{i} \partial \xi_{j}}\right)_{T, \mathbf{x}, \xi^{\circ}} i, j=s+1, s+2, \ldots
\end{gathered}
$$

It is assumed that a stationary state of thermodynamic system near the equilibrium state is steady, so that matrix $S_{l k}\left(\xi_{1}^{\circ}, \xi_{2}^{\circ}, \ldots, \xi_{s}^{\circ}\right)$ in expression (21) is positive definite; entropy, as a function of internal variables, in the fixed stationary state has a maximum. The properties of matrix $S_{l k}$ in a stationary point, which is far from equilibrium, remain not certain.

Relation (21) allows us to write an expression for thermodynamic forces in a point near the stationary state:

$$
\begin{aligned}
\Xi_{j}(\xi)= & -T \frac{\partial S}{\partial \xi_{j}}=\Xi_{j}\left(\xi^{\circ}\right) \\
& +T S_{j l}\left(\xi_{l}-\xi_{l}^{\circ}\right)+\cdots, \quad j=1,2, \ldots, s .
\end{aligned}
$$

The first terms of the expansion of the thermodynamic forces are constant. In the situations close to the equilibrium state, in the simplest approach, thermodynamic forces are connected linearly with internal variables:

$$
\Xi_{j}=-T \frac{\partial S}{\partial \xi_{j}}=T S_{j k} \xi_{k}+\cdots
$$

Let us remember that, in the state of equilibrium, values of internal variables are considered to be zero, and thermodynamic forces $\Xi_{j}$ disappear.

4.3. Production of Entropy. In a stationary nonequilibrium state, values of temperature and all constitutive variables of the thermodynamic system are constant. Values of thermodynamic functions of system, including entropy, also are constant; however, there is production of entropy inside the system and corresponding decrease in entropy of the system, due to fluxes of heat and/or substances, so that one can write

$$
\frac{d_{i} S}{d t}=-\frac{d_{e} S}{d t} .
$$


Equations (17) and (22) allow us to write an expansion of function of production of entropy (11) near a steady-state point:

$$
\begin{aligned}
\frac{d_{i} S}{d t}= & -\frac{1}{T} \sum_{j=1}^{s} \Xi_{i} J_{i} \\
& +\frac{1}{T} \sum_{j, l=1}^{s}\left(-T S_{j l} J_{j}+\Xi_{j} R_{j l}\right)\left(\xi_{l}-\xi_{l}^{\circ}\right) \\
& +\sum_{j, k, l=1}^{s}\left(S_{j k} R_{j l}+\Xi_{j} \frac{\partial R_{j k}}{\partial \xi_{l}}\right)\left(\xi_{k}-\xi_{k}^{\circ}\right)\left(\xi_{l}-\xi_{l}^{\circ}\right) \\
& +\sum_{j, k, l=s+1}^{\infty} S_{j k} R_{j l} \xi_{k} \xi_{l}+\cdots
\end{aligned}
$$

Values of all matrixes are determined in the considered steady-state point $\xi_{1}^{\circ}, \xi_{2}^{\circ}, \ldots, \xi_{s}^{\circ}$.

In the simplest approximation, expression for production of entropy can be written as

$$
\frac{d_{i} S}{d t}=-\frac{1}{T} \sum_{j=1}^{s} \Xi_{j} J_{j}
$$

This expression represents production of entropy in the conventional form as the sum of products of fluxes and thermodynamic forces. Equation (26) is considered as one of the basic statements of nonequilibrium thermodynamics [13 ]. The emerging of the sign "minus" in expression (26) is connected with the fact that signs of the fluxes are taken opposite to signs of the forces (the internal variables). One can note, that representation (26) is valid only for steady-state situations and for small deviations from equilibrium state.

Comparison of (9) and (26) for a stationary state, when relation (24) is valid, determines that quantity $K_{j}$ in (9) coincides with thermodynamic force, $K_{i}=\Xi_{i}, i=1,2, \ldots, s$.

4.4. On the Criterion of Stability of Stationary States. Expression (25) shows that, in a steady-state point, production of entropy, as a function of the internal variables of complexity, has no peculiar points. However, if the steady-state point is fixed $\left(\xi_{l}=\xi_{l}^{\circ}, l=1,2, \ldots s\right)$, expression (25) takes the form

$$
\frac{d_{i} S}{d t}=-\frac{1}{T} \sum_{j=1}^{s} \Xi_{i} J_{i}+\sum_{j, k, l=s+1}^{\infty} S_{j k} R_{j l} \xi_{k} \xi_{l}+\cdots .
$$

The behaviour of production of entropy in vicinity of a steady-state point is determined by terms of the second order with respect to all internal variables, excepting the complexity variables. The terms of the second order comprise a square form with the matrix that is a product of two matrixes $R_{j k}$ and $S_{l k}$, which are calculated in the steady-state point and depend, apart from temperature and constitutive variables, on the complexity internal variables. In the equilibrium point and, assumingly, in the steady-state points near to the equilibrium state the matrixes are positive definite, so that production of entropy has a minimum, which confirms the validity of the Prigogine's principle of a minimum of production of entropy [1-3]. In the points, that are far from the equilibrium point, the matrixes $R_{j k}$ and $S_{l k}$ are not necessarily positive definite, so that stability of the system can be connected with a maximum of production of entropy, as it is stated by some investigators $[11,12]$.

4.5. The Relation between Fluxes and Thermodynamic Forces. Now, with help of (18) and (23), we can write expression (26) for production of entropy in other form as

$$
\frac{1}{T} \sum_{j, k=1}^{s} \Xi_{j} R_{j k} \xi_{k}=-\sum_{j, k=1}^{s} S_{j k} \xi_{k} J_{j}
$$

The written equation, by virtue of the supposed arbitrariness and independence of internal variables, is followed by a relation between fluxes and thermodynamic forces:

$$
\frac{1}{T} \sum_{j=1}^{s} \Xi_{j} R_{j k}=-\sum_{j=1}^{s} S_{j k} J_{j}
$$

This relation can be rewritten as

$$
J_{i}=-L_{i k} \Xi_{k}, \quad L_{i k}=\frac{1}{T} R_{i l} S_{l k}^{-1} .
$$

Considering linear approach, the components of matrixes $R_{j k}$ and $S_{l k}$ are constants, but in more general case it is necessary to consider them as functions of internal variables. By virtue of definition, the matrix $L$ is positive definite.

The relation between fluxes and thermodynamic forces in linear approximation are fundamental relation of the nonequilibrium thermodynamics [1-3]. We have shown that these parities are consequence of the main principles of thermodynamics and valid under two conditions: first, deviations from an equilibrium state are small, and second, the state is stationary.

Let us note in addition that there is a statement [1-3] for the matrix $L$ to be symmetric or antisymmetric:

$$
L_{i j}= \pm L_{j i} \text {. }
$$

For the proof of this statement, to which usually refer as to Onsager principle, one has to address the other principles and some assumptions considered in the following section.

4.6. Symmetry of Kinetic Coefficients. The proof of the symmetry of kinetic coefficients is based on the property of invariance of correlations of fluctuations of various quantities with respect to reversion of time, which is fair for equilibrium situations [1-3, 13]. Being interested in stationary states, it is possible to consider fluctuations of internal variables near their stationary values and to assume that time correlations of random deviations of internal variables also are invariant with respect to reversion of time. In other words, for correlations of various quantities, it is possible to write a relation

$$
\begin{aligned}
& \left\langle\left(\xi_{i}-\xi_{i}^{\circ}\right)_{t}\left(\xi_{k}-\xi_{k}^{\circ}\right)_{0}\right\rangle \\
& \quad= \pm\left\langle\left(\xi_{k}-\xi_{k}^{\circ}\right)_{t}\left(\xi_{i}-\xi_{i}^{\circ}\right)_{0}\right\rangle .
\end{aligned}
$$


The "minus" sign arises in the case, when the internal variable itself changes the sign at the reversion of time.

Further, one can take advantage of the equation of evolution (16), which, with use of expression for thermodynamic force (22) and definition of a matrix of kinetic coefficients (30), can be presented in the form

$$
\frac{d\left(\xi_{i}-\xi_{i}^{\circ}\right)}{d t}=-L_{i l}\left(\Xi_{l}(\xi)-\Xi_{l}\left(\xi^{\circ}\right)\right) .
$$

This equation allows one, after differentiation of relation (32), to write down

$$
\begin{aligned}
& L_{i l}\left\langle\left(\Xi_{l}(\xi)-\Xi_{l}\left(\xi^{\circ}\right)\right)\left(\xi_{k}-\xi_{k}^{\circ}\right)_{0}\right\rangle \\
& \quad= \pm L_{k l}\left\langle\left(\Xi_{l}(\xi)-\Xi_{l}\left(\xi^{\circ}\right)\right)\left(\xi_{i}-\xi_{i}^{\circ}\right)_{0}\right\rangle .
\end{aligned}
$$

By virtue of supposed independence of deviations of various variables from stationary values, the required relation (31) follows the equation written previously.

Expansion of the principle of invariance of correlations with respect to reversion of time for stationary situations allows proving the principle of symmetry of kinetic coefficients.

4.7. A Simple Example. Let us consider a portion of matter in a small volume $\Delta x \Delta y \Delta z$ as a thermodynamic system. In addition to the cases, when a stationary state is maintained by fluxes of heat and particles separately [7], let us consider the simultaneous action of these factors. We can assume that streams of heat and a substance are moving along the $x$-axis, so that

$$
J_{\mathrm{T}}=\frac{1}{\Delta y \Delta z} \frac{\Delta Q}{\Delta t}, \quad J_{\mathrm{C}}=\frac{1}{\Delta y \Delta z} \frac{\Delta m}{\Delta t} .
$$

The fluxes of heat and substance are contributing independently in the entropy of the volume. The flux of heat, according to (8), determines the change of density of entropy in the volume:

$$
\frac{d_{\text {heat }} S}{d t}=\frac{1}{\Delta x}\left(\frac{J_{\mathrm{T}}}{T}-\frac{J_{\mathrm{T}}}{T+\Delta T}\right) \approx \frac{J_{\mathrm{T}}}{T^{2}} \nabla_{x} T
$$

In a similar way, one can define the change of density of entropy due to the flux of the substance:

$$
\frac{d_{\text {subs }} S}{d t}=\frac{J_{C}}{T} \frac{\mu(c+\Delta c)-\mu(c)}{\Delta x} \approx \frac{J_{C}}{T} \frac{\partial \mu}{\partial c} \nabla_{x} c .
$$

The system is assumed to be characterised by chemical potential $\mu(c)$, which depends on concentration $c$.

It is convenient to introduce special symbols for the gradients of temperature and concentration, which can be considered to be internal complexity variables:

$$
\xi_{\mathrm{T}}=\nabla_{x} T=\frac{\Delta T}{\Delta x}, \quad \xi_{\mathrm{C}}=\nabla_{x} c=\frac{\Delta c}{\Delta x} .
$$

Thus, the change of density of entropy, due to the external fluxes of heat and substance, can be written as follows:

$$
\frac{d_{e} S}{d t}=\frac{J_{\mathrm{T}}}{T^{2}} \xi_{\mathrm{T}}+\frac{J_{\mathrm{C}}}{T} \frac{\partial \mu}{\partial c} \xi_{\mathrm{C}}
$$

The fluxes and gradients are connected with each other locally. It is well known [1-3], as an empirical fact, that, at simultaneous presence of the thermal and diffusion gradients, $\xi_{\mathrm{T}}$ and $\xi_{\mathrm{C}}$ cross-effects are observed; a gradient of temperature induces a flux of the substance and vice versa, a gradient of concentration induces a flux of heat, so that according to the last equation from (18), we record a linear parity connecting the fluxes and gradients for the considered system:

$$
\begin{aligned}
& J_{\mathrm{T}}=-R_{\mathrm{TT}} \xi_{\mathrm{T}}-R_{\mathrm{TC}} \xi_{\mathrm{C}}, \\
& J_{\mathrm{C}}=-R_{\mathrm{CT}} \xi_{\mathrm{T}}-R_{\mathrm{CC}} \xi_{\mathrm{C}} .
\end{aligned}
$$

These equations allow one to write the internal production of entropy, according to (26), in the form

$$
\begin{aligned}
\frac{d_{i} S}{d t}=\frac{1}{T}\left[\left(R_{\mathrm{TT}} \xi_{\mathrm{T}}+R_{\mathrm{TC}} \xi_{\mathrm{C}}\right) \Xi_{\mathrm{T}}\right. \\
\left.+\left(R_{\mathrm{CT}} \xi_{\mathrm{T}}+R_{\mathrm{CC}} \xi_{\mathrm{C}}\right) \Xi_{\mathrm{C}}\right] .
\end{aligned}
$$

In a steady-state situation, the thermodynamic state of the system does not change, so that (24) is valid, and taking relation (39) into account, one has another expression for production of entropy:

$$
\frac{d_{i} S}{d t}=-\frac{J_{\mathrm{T}}}{T^{2}} \xi_{\mathrm{T}}-\frac{J_{\mathrm{C}}}{T} \frac{\partial \mu}{\partial c} \xi_{\mathrm{C}} .
$$

Comparing (41) and (42), one finds the relations between fluxes and thermodynamic forces for a stationary case:

$$
\begin{aligned}
& J_{\mathrm{T}}=-T\left(R_{\mathrm{TT}} \Xi_{\mathrm{T}}+R_{\mathrm{CT}} \Xi_{\mathrm{C}}\right), \\
& J_{\mathrm{C}}=-\left(\frac{\partial \mu}{\partial c}\right)^{-1}\left(R_{\mathrm{TC}} \Xi_{\mathrm{T}}+R_{\mathrm{CC}} \Xi_{\mathrm{C}}\right) .
\end{aligned}
$$

The condition of symmetry of kinetic coefficients (31) is followed by an equation:

$$
\frac{1}{T} R_{\mathrm{TC}}=\frac{\partial \mu}{\partial c} R_{\mathrm{CT}} .
$$

Entropy of the system is defined by formula (6), which, in considered case, takes the form

$$
S-S_{0}=-\frac{1}{T} \int_{0}^{\nabla_{x} T} \int_{0}^{\nabla_{x} c}\left(\Xi_{\mathrm{T}} d \xi_{\mathrm{T}}+\Xi_{\mathrm{C}} d \xi_{\mathrm{C}}\right) .
$$

The thermodynamic forces $\Xi_{\mathrm{T}}$ and $\Xi_{\mathrm{C}}$, as functions of complexity variables, can be found from (40) and (43). After the calculation of the integrals, one gets a simple formula for entropy as a function of internal variables:

$$
S-S_{0}=-\frac{1}{2 T}\left(\frac{1}{T}\left(\nabla_{x} T\right)^{2}+\frac{\partial \mu}{\partial c}\left(\nabla_{x} c\right)^{2}\right) .
$$

It is remarkable that the condition of integrability and existence of entropy of the system appears to be identical to Onsager's relation, that is, equality of the nondiagonal components of a matrix in (43). 


\section{Conclusion}

The usage of internal variables, playing a distinctive role in the description of nonequilibrium states of the system, allows us to develop a generalized view on the principle of nonequilibrium thermodynamics. In this paper, in line with the paper [7], the author aspired to show that the thermodynamics of nonequilibrium states could be formulated so consistently, as the thermodynamics of equilibrium states. At least, considering the situations close to equilibrium and using the simplest approach, we have obtained the fundamental relations of non-equilibrium thermodynamics. One can note the most essential features of our description.

(i) The reference to local equilibrium is not used. On the contrary, it is supposed, that local nonequilibrium exists. A set of internal variables $\xi_{1}, \xi_{2}, \ldots$ are introduced to describe deviation of the system from equilibrium.

(ii) While formulating the law of conservation of energy, the work connected with internal variables is considered, thus, one has to distinguish between the total internal energy $U(T, \mathbf{x}, \boldsymbol{\xi})$ and internal thermal energy $E(T, \mathbf{x})$ of the system. It allows to introduce entropy $S$ for non-equilibrium states of thermodynamic system by (4), which keeps the meaning of entropy unchanged. As a consequence of this, temperature in any situation is defined through thermodynamic functions of the system as $T=(\partial E / \partial S)_{\mathbf{x}}$ or $T=$ $(\partial U / \partial S)_{\mathbf{x}, \boldsymbol{\xi}}$.

(iii) The second principle of thermodynamics may be formulated as impossibility of fulfilment of positive work by internal variables (Section 2.3). This formulation is equivalent to conventional formulations but excludes a flavour of mystique from concept of entropy.

(iv) Relation (5), that connects a change of entropy of a thermodynamic system with fluxes of heat and substance and also with change of internal variables, is valid for the cases of open systems and irreversible processes. It is a generalisation of Prigogine's relation, given by him for the systems with chemical reactions [1, Equation 3.52].

(v) The known linear relations of nonequilibrium thermodynamics (in particular, the parity between forces and the fluxes) follow from the general principles for the cases of steady-state processes running in the region of small deviations from equilibrium.

One can see that all known results of linear non-equilibrium thermodynamics follow the presented approach; however, the theory is open for nonlinear generalisations.

\section{Endnotes}

1. Perhaps, the first who used internal variables for the consecutive formulation of thermodynamics was Leontovich [5]. The first Russian edition of "Introduction to Thermodynamics," based on his prewar- and war-time lectures, has appeared, as a separate book, in 1950.
2. Temperature is usually measured in degrees, but, according to relationship with other physical quantities, it is energy. To omit the factor of transition from degrees to energy, it is convenient to agree that the absolute temperature $T$ is measured in energy units as it was proposed by Landau and Lifshitz [13] and used here through the paper.

3. We do not include here into consideration the fluctuations in thermodynamic systems (local deviations of density, temperatures, concentration of substances, and other quantities from equilibrium values) which can be treated as huge number of uncontrollable internal variables.

4. In nonequilibrium situations, work of the system is not equal to the work of the environment on the system [14]. To balance the inner and outer forces, one needs, as it was shown by Gujrati [14], in some internal variables.

5. The alternative theory $[4,6]$ of non-equilibrium in terms of internal variables does not distinguish between the total and internal energies, thus, misinterpreting the role of internal variables.

6. The definition of entropy in the alternative theory $[4,6]$ of non-equilibrium in terms of internal variables does not include fluxes of substances (the last term in (5)), thus, failing to reproduce the Prigogine's result. Moreover, one needs in the omitted term to consider the processes of diffusion correctly, as was discussed earlier [7].

7. There are many important exclusions from this rule. A description of thermodynamic systems with oscillating chemical reactions, for example, requires matrix $R_{i j}$ not to be positive definite.

8. It is known, that such combination (transformation) of variables can be chosen that the equation of dynamics (16) for these variables gets the form of the equation of a relaxation:

$$
\frac{d \xi_{i}}{d t}=-\frac{1}{\tau_{i}} \xi_{j}, \quad i=1,2, \ldots, s,
$$

where $\tau_{i}=\tau_{i}\left(T, x_{1}, x_{2}, \ldots, x_{n}\right)$ is a time of relaxation of corresponding variable. Changing under internal laws of the movement, the agitated internal variables trend to their unique equilibrium value, so that the system is moving to its equilibrium state.

\section{Acknowledgment}

The author is grateful to the anonymous reviewers of the paper for their helpful and constructive comments.

\section{References}

[1] I. Prigogine, Introduction To Thermodynamics of Irreversible Processes, Edited by C. C. Thomas, Sprinfild, Geneseo, Ill, USA, 1955. 
[2] S. R. de Groot and P. Mazur, Non-Equilibrium Thermodynamics, NorthHolland, Amsterdam, The Netherlands, 1962.

[3] D. Kondepudi and I. Prigogine, Modern Thermodynamics. From Heat Machines to Dissipative Structures, John Wiley \& Sons, Chichester, UK, 1999.

[4] W. Muschik, "Why so many "schools" of thermodynamics?" Forschung im Ingenieurwesen, vol. 71, no. 3-4, pp. 149-161, 2007.

[5] M. A. Leontovich, Introduction to Thermodynamics. Statistical Physics, Nauka, Glavnaja redaktzija fisiko-matematicheskoi literatury, Moscow, Russia, 1983.

[6] G. A. Maugin and W. Muschik, "Thermodynamics with internal variables Part I. general concepts," Journal of Non-Equilibrium Thermodynamics, vol. 19, no. 3, pp. 217-249, 1994.

[7] V. N. Pokrovski, "Extended thermodynamics in a discrete-system approach," European Journal of Physics, vol. 26, no. 5, pp. 769-781, 2005.

[8] J. Kestin, "the local-equilibrium approximation," Journal of NonEquilibrium, vol. 18, no. 4, pp. 360-379, 1993.

[9] W. Muschik, "Comment to J. Kestin: internal variables in the local-equilibrium approximation," Journal of Non-Equilibrium Thermodynamics, vol. 18, pp. 380-388, 1993.

[10] R. L. Stratonovich, Nonlinear Nonequilibrium Thermodynamics, Springer, Heidelberg, Germany, 1994.

[11] A. Kleidon, "Nonequilibrium thermodynamics and maximum entropy production in the Earth system: applications and implications," Naturwissenschaften, vol. 96, no. 6, pp. 653-677, 2009.

[12] K. Hackl and F. D. Fischer, "On the relation between the principle of maximum dissipation and inelastic evolution given by dissipation potentials," Proceedings of the Royal Society A, vol. 464, no. 2089, pp. 117-132, 2008.

[13] L. D. Landau and E. M. Lifshitz, Statistical Physics, vol. 1, Pergamon Press, Oxford, UK, 3rd edition, 1986.

[14] P. D. Gujrati, "Nonequilibrium thermodynamics. Symmetric and unique formulation of the first law, statistical definition of heat and work, adiabati theorem and the fate of the Clausius inequality: a microscopi view," http://arxiv.org/abs/1206.0702. 

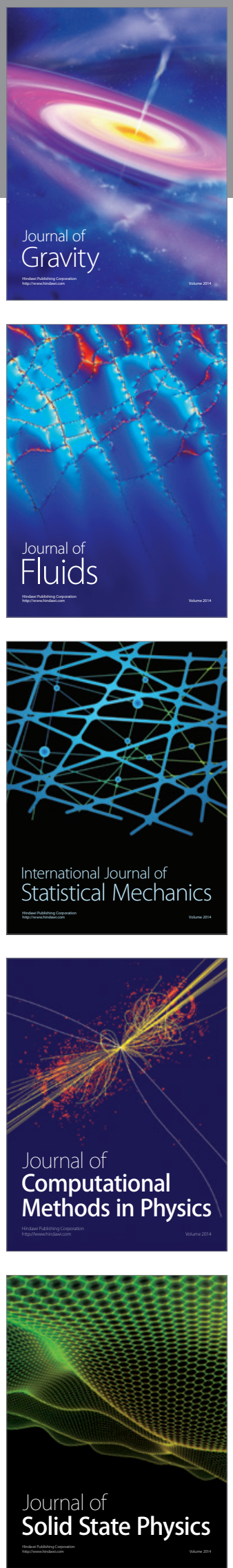

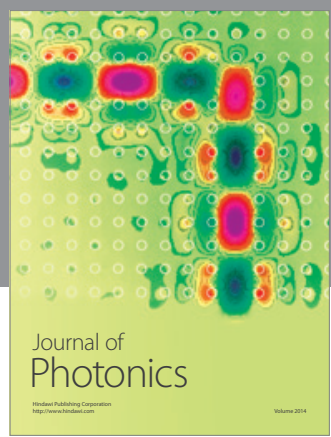

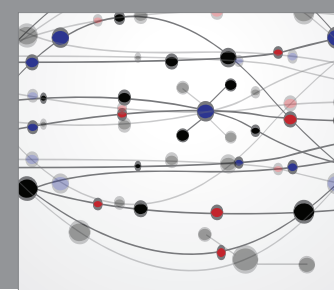

The Scientific World Journal

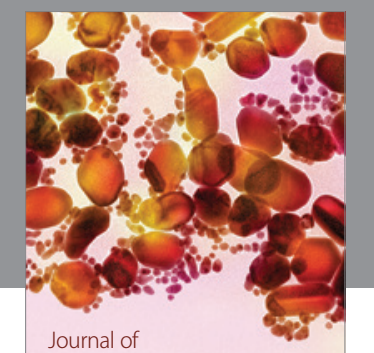

Soft Matter
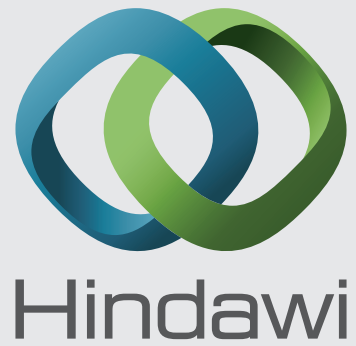

Submit your manuscripts at

http://www.hindawi.com
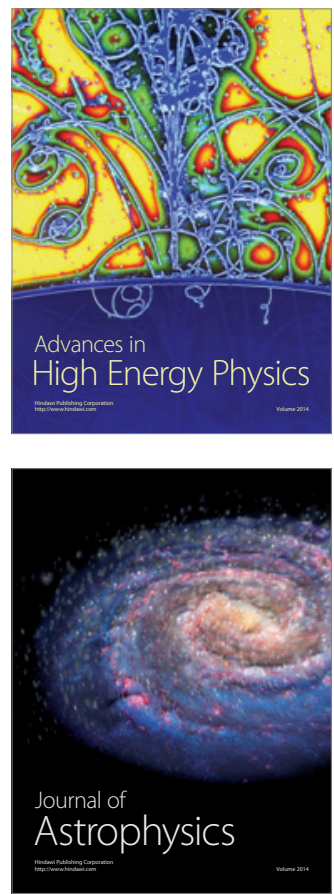
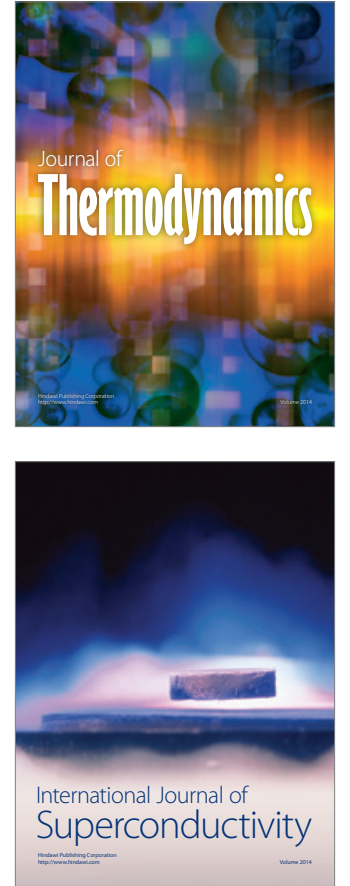
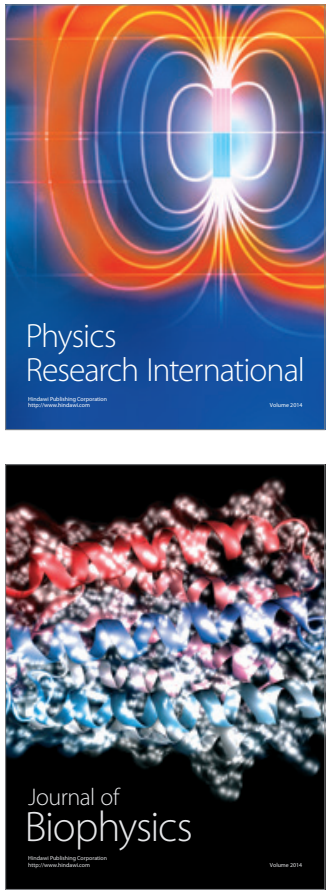
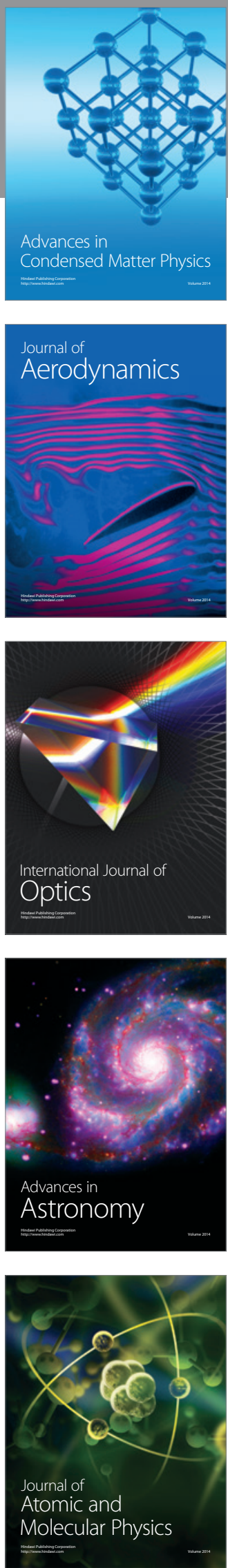